\title{
Electroencephalogram assessment of mental fatigue in visual search
}

\author{
Xiaoli Fan ${ }^{\mathrm{a}}$, Qianxiang Zhou ${ }^{\mathrm{a},{ }^{*}}$, Zhongqi Liu ${ }^{\mathrm{a}}$ and Fang Xie ${ }^{\mathrm{b}}$ \\ ${ }^{a}$ School of Biological Science and Medical Engineering, Beihang University, No. 37, Xueyuan Road, \\ Haidian District, Beijing 100191, China \\ ${ }^{b}$ China North Vehicle Research Institute, Beijing 100072, China
}

\begin{abstract}
Mental fatigue is considered to be a contributing factor responsible for numerous road accidents and various medical conditions and the efficiency and performance could be impaired during fatigue. Hence, determining how to evaluate mental fatigue is very important. In the present study, ten subjects performed a long-term visual search task with electroencephalogram recorded, and self-assessment and reaction time (RT) were combined to verify if mental fatigue had been induced and were also used as confirmatory tests for the proposed measures. The changes in relative energy in four wavebands $(\delta, \theta, \alpha$, and $\beta)$, four ratio formulas $[(\alpha+\theta) / \beta, \alpha / \beta,(\alpha+\theta) /(\alpha+\beta)$, and $\theta / \beta]$, and Shannon's entropy (SE) were compared and analyzed between the beginning and end of the task. The results showed that a significant increase occurred in alpha activity in the frontal, central, posterior temporal, parietal, and occipital lobes, and a dip occurred in the beta activity in the pre-frontal, inferior frontal, posterior temporal, and occipital lobes. The ratio formulas clearly increased in all of these brain regions except the temporal region, where only $\alpha / \beta$ changed obviously after finishing the 60 -min visual search task. SE significantly increased in the posterior temporal, parietal, and occipital lobes. These results demonstrate some potential indicators for mental fatigue detection and evaluation, which can be applied in the future development of countermeasures to fatigue.
\end{abstract}

Keywords: Mental fatigue, EEG, ratio formulas, entropy, visual search

\section{Introduction}

Sustained mental efficiency in cognitively demanding activities leads to the exhaustion of cognitive resources and to a state of so-called "mental fatigue" [1]. Among many factors, mental fatigue is considered to be a contributing factor responsible for numerous road accidents or other potentially adverse incidents [2]. In addition, fatigue can lead to various diseases such as cardiovascular disease, epileptic seizures, and karoshi [3]. Its general symptoms are non-specific: generally it manifests in the form of drowsiness, weakness, dizziness or queasiness. Fatigue can lead to distractibility and provokes lapses in information processing [4]. In other words, when one persists in continuing the current work as normal, efficiency and performance can be reduced during fatigue [5]. Therefore, the detection and evaluation of fatigue will be helpful for the early assessment of mental fatigue levels. A considerable

\footnotetext{
* Address for correspondence: Qianxiang Zhou, School of Biological Science and Medical Engineering, Beihang University, No. 37, Xueyuan Road, Haidian District, Beijing 100191, China. Tel.: 010-82338696; Fax: 010-82338696; Email: zqxg@buaa.edu.cn.
} 
number of studies have been carried out to evaluate mental fatigue by various measures, including both subjective and objective evaluations. Subjective evaluation is mainly in the form of questionnaires, such as the Piper Fatigue Scale or the Epworth Sleepiness Scale [6, 7]. However, subjective evaluation is easily affected by individual difference. Objective evaluation includes measures based on reaction time, ocular parameters, and physiological variables like the Electroencephalogram (EEG), Electrooculogram (EOG), Electromyogram (EMG) or Electrocardiogram (ECG) [4].

Among physiological variables, EEG is considered to be one of the most predictive and reliable indicators of mental fatigue [8,9], and it may also become a promising fatigue countermeasure approach [10]. EEG recorded from the human scalp is mainly composed of four frequency wavebands, which are $\delta(0.5-4 \mathrm{~Hz}), \theta(4-8 \mathrm{~Hz}), \alpha(8-16 \mathrm{~Hz})$, and $\beta(16-32 \mathrm{~Hz})$, and it is closely related to psychological and physical activities. Studies show that the EEG power shifts toward low-frequency bands when a subject is experiencing fatigue [11-13]. Alpha waves are considered a reliable determinate of the early stages of fatigue and are widely monitored in many fatigue studies $[14,15]$. Waard and Brookhuits [16] indicated that EEG energy ratio, $(\alpha+\theta) / \beta$, tended to fall as a task continued. Meanwhile, Jap [17] showed that three EEG energy ratios, $\alpha / \beta,(\alpha+\theta) /(\alpha+\beta)$, and $\theta / \beta$, increased with an increase in fatigue. In addition, entropy is a measure of information content and is believed to be a possible indicator of fatigue. However, these effects show a high variability and largely depend on the experimental design and the studied subjects, and different activities may lead to different magnitudes or frequencies in EEG recordings.

In most fatigue studies, the methods used to induce fatigue are either through sleep deprivation or the performance of long-term, tedious, and repetitive tasks. However, both fatigue-inducing methods produce similar decrements in performance, and May and Baldwin [18] deduced that the evaluation methods may be applied for all types of mental fatigue. In the present study, a long-term visual search task, which needed sustained attention, was used to induce fatigue. Mental fatigue is a complicated process comprising physical, psychosocial, and behavioral factors; thus, it is not reliable and comprehensive enough to use a single-parameter measure to evaluate mental fatigue [13]. Therefore, we used a combination of several measures. The self-assessment of fatigue levels, reaction time (RT) and accuracy in performing a visual search task were used to verify whether mental fatigue had been induced and were also used as confirmatory tests for the proposed measures. In the present study, we characterized EEG signals in the wavelet domain using several measures, including power parameters in four wavebands $(\delta, \theta, \alpha$, and $\beta)$, four ratio formulas $[(\alpha+\theta) / \beta, \alpha / \beta,(\alpha+\theta) /(\alpha+\beta)$, and $\theta / \beta]$, and Shannon's entropy (SE).

\section{Materials and methods}

\subsection{Study subjects}

Ten male college students from Beihang University between the ages of 20 and 28 participated in this experiment voluntarily. All reported having normal or corrected to normal visual acuity as well as normal sleep-wake habits. None had medical contraindications such as drug abuse or mental disorders, including depression or anxiety. Before the experiment, the subjects slept more than $7 \mathrm{~h}$ each and were asked to abstain from consuming alcohol, tea, or other caffeinated substances and smoking.

\subsection{Visual search task design}




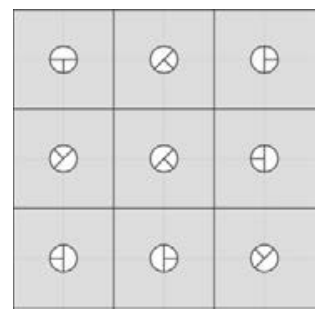

Fig. 1. Visual search interface.

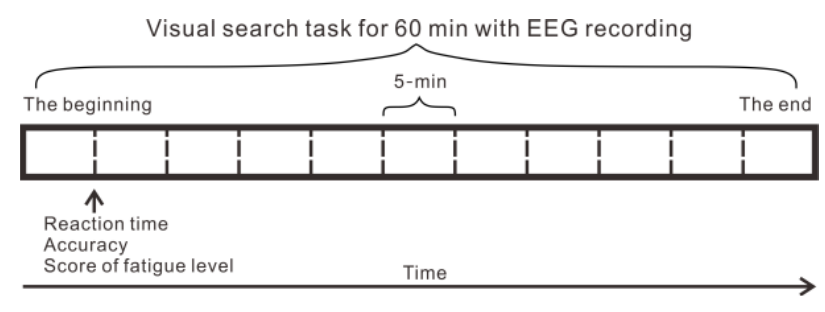

Fig. 2. Experimental design.

The experiment was conducted using custom-made software that provided the subjects with a visual search task. The visual search picture was divided into a $3 \times 3$ grid with a symbol in each square (Figure 1). Among the symbols, $\otimes$ was the target stimuli, while $\odot, \oplus, \ominus, \odot, \otimes, \otimes$, and $\oslash$ represented distractive stimuli. The visual search picture changed once every $3 \mathrm{~s}$, and the symbols randomly appeared in different squares of the picture. For each search picture, there were two situations: having target or no target, and each situation's probability was $50 \%$.

When the test began and search pictures appeared, subjects had to quickly choose among two actions: (1) If the target was discovered, the left mouse button must be pressed as quickly as possible; (2) If no target is seen, the right mouse button should be immediately pressed. During the experiment, the software recorded the accuracy and reaction time.

\subsection{Experimental procedure}

The experiment was conducted from 9:00 AM to 10:00 AM or 3:00 PM to 4:00 PM in a quiet, sound-attenuated, electrically shielded room. The experimental process is shown in Figure 2. The subjects were allowed to have approximately 5 min of practice using the software, after which they were asked to perform the test for $60 \mathrm{~min}$ as quickly and as correctly as possible. In order to evaluate the visual search performance over the course of the experiment, the task was divided into twelve 5min sections. When each section was completed, the software recorded the average reaction time and accuracy of this section automatically. Meanwhile, the subjects scored their fatigue levels in written form according to the rules shown in Table 1 and then started the next section as soon as possible. Simultaneously, EEG was recorded.

\subsection{EEG recording and processing}

A NeuroScan 64 channel system with the international 10-20 lead system was used to record the EEG signal. The original signal was amplified by a Synamps2/RT amplifier and was converted from analog to digital (A/D). The EEG signal was characterized for eight brain regions (with corresponding

Table 1

Subjective fatigue evaluation

\begin{tabular}{llll}
\hline Level & Fatigue state & Symptom & Score \\
\hline 1 & Alert & Focused attention, highly aroused & 0 \\
2 & Awake & Sober, without obvious feeling & $1-2$ \\
3 & Mild fatigue & Mild eyestrain and stinging & $3-4$ \\
4 & Moderate fatigue & Moderate eyestrain and stinging, moderately dizzy, mild nausea, and headache & $5-7$ \\
5 & Severe fatigue & Severe eyestrain and dizziness, moderate or severe headache, nausea and vomiting & $8-10$ \\
\hline
\end{tabular}


electrodes), including the pre-frontal (Fp1 and Fp2), inferior frontal (F7 and F8), frontal (F3, F4, and $\mathrm{Fz})$, temporal (T7 and $\mathrm{T} 8)$, central $(\mathrm{C} 3, \mathrm{C} 4$, and $\mathrm{Cz}$ ), posterior temporal $(\mathrm{P} 7$ and $\mathrm{P} 8)$, parietal (P3 and $\mathrm{P} 4)$, and occipital $(\mathrm{O} 1, \mathrm{O} 2$, and $\mathrm{Oz})$ lobes. Meanwhile, EOG channels were also recorded and used to distinguish the blinks artifacts from raw signals. An average mastoid reference (M1 and M2) was computed offline and subtracted from all brain channels. To obtain an effective EEG signal, the impedance of the selected channels was adjusted below $5 \mathrm{~K} \Omega$ before recording. The signal was sampled at $500 \mathrm{~Hz}$ with a $0.05-200 \mathrm{~Hz}$ band-pass filter.

There was a considerable amount of noise named artifacts in the raw EEG signal, possibly originating from biological and technical artifacts inherent to measurement conditions, such as eye blinks, eye movements, respiration, heartbeat, muscle activity, and power interference of $50 \mathrm{~Hz}$. Signals with amplitudes greater than $50 \mu \mathrm{V}$ in EEG raw signals were considered to be artifacts and were thus removed [19]. The extended Independent Component Analysis (ICA) was used to remove the artifacts corresponding to eye blinks and eye movements among others. Noise caused by unnecessary movement was manually removed. The EEG signal was processed through a band-pass filter of $0.5-40 \mathrm{~Hz}$.

For the non-stationary characteristics of the EEG signal, wavelet packet analysis was used to detect EEG rhythms $(\delta, \theta, \alpha$, and $\beta)$ and to analyze the EEG power spectrum. The selected EEG signals were processed by wavelet packet analysis with six-octave wavelet decomposition. The dB4 was considered to be the most suitable function and was chosen as the mother wavelet. Sixty four frequency components were obtained after the decomposition, and the smallest frequency resolution is evaluated as Eq. (1), where $f_{s}$ represents the sampling frequency.

$$
\Delta \mathrm{f}=\frac{1}{2^{6}} \times \frac{f_{s}}{2}=3.906 \mathrm{~Hz}
$$

We reconstructed the wavelet decomposition coefficients and extracted each frequency waveband. Subsequently, the energy of the four frequency wavebands was expressed as Eq. (2), where $j$ represents the different frequency wavebands $(\delta, \theta, \alpha$, and $\beta), C_{j}$ is the wavelet coefficient that formed the corresponding frequency waveband, and $\mathrm{L}$ is the total number of coefficients.

$$
E_{j}=\sum_{k=1}^{L} C_{j}(k)^{2} \quad(j=1,2,3,4)
$$

The relative energy of each waveband can be expressed as Eq. (3):

$$
R E_{j}=\frac{E_{j}}{\sum_{k=1}^{4} E_{k}} \quad(j=1,2,3,4)
$$

Ratios $(\alpha+\theta) / \beta, \alpha / \beta,(\alpha+\theta) /(\alpha+\beta)$, and $\theta / \beta$ were calculated and used as indicators to evaluate fatigue. Meanwhile, SE, which is a disorder quantifier in the wavelet domain, was also proposed in this paper as an indicator of fatigue [20], and it is expressed as in Eq. (4):

$$
\mathrm{SE}=-\sum_{j=1}^{4} R E_{j} \cdot \log \left(R E_{j}\right)
$$

\section{Results}

\subsection{Self-report}




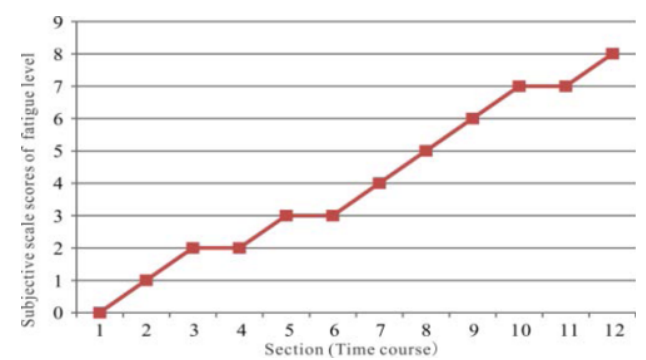

Fig. 3. Subjective scale scores of fatigue level during the visual searching for a single study subject.

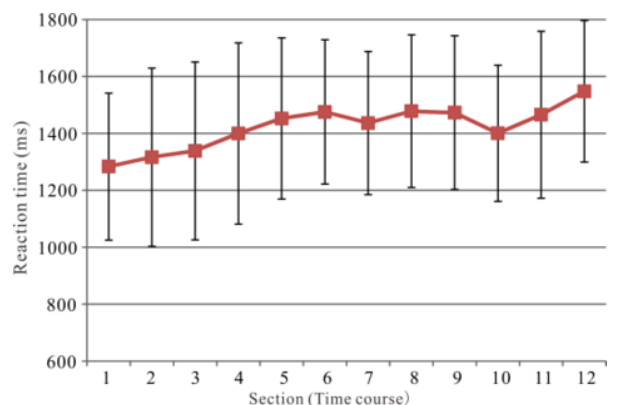

(a) Mean accuracy

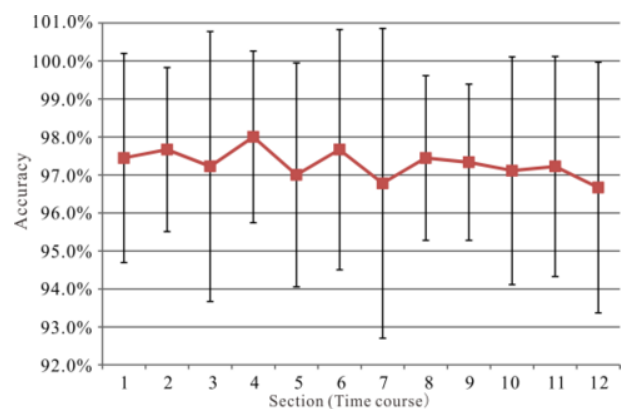

(b) Mean reaction time

Fig. 4. Change in performance over time during visual searching.

Figure 3 shows one subject's subjective scale scores of mental fatigue during the visual search task based on the questionnaire (Table 1). It demonstrates that the fatigue level increased as time went on, a pattern that was also seen in other subjects. Most subjects felt that they began to have difficulty in concentrating on the task after approximately $25 \mathrm{~min}$, and felt anxious, irritable, and close to an emotional breakdown by the end. Thus, mental fatigue was clearly induced by $60 \mathrm{~min}$ of performing a continuous visual search task.

\subsection{RT and accuracy}

Changes over time in task performance are shown in Figure 4. One-way repeated-measure ANOVA with time was used to analyze the effect of time-on-task for both RT and accuracy. There was a significant main effect of time on $\mathrm{RT}(\mathrm{F}=3.101, \mathrm{P}=0.046)$ but no effect on accuracy $(\mathrm{F}=1.034, \mathrm{P}=$ 0.397). In previous studies, RT measures have been associated with fatigue from sustained mental workload [21, 22]. In Figure 4, RT increases in a linear fashion from the beginning to the end of task performance $\left(\mathrm{R}^{2}=0.69\right)$, while there is a mild decline in accuracy. Therefore, RT measures can be used in fatigue studies as a primary behavioral outcome. These findings also demonstrated that the subjects were mentally fatigued after the task, suggesting the validity of using $60 \mathrm{~min}$ of visual searching as a fatigue inducement technique.

\subsection{EEG data}

EEG data from 5 min intervals at the beginning ( $1^{\text {st }}$ section $)$ and end $\left(12^{\text {th }}\right.$ section $)$ of the task were chosen for analysis, and a paired t-test was conducted. Table 2 shows the results of the visual search task in all brain regions for all evaluation parameters. We observed that clear changes occurred in the 
Table 2

T-test results for delta, theta, alpha, beta, $(\alpha+\theta) / \beta, \alpha / \beta,(\alpha+\theta) /(\alpha+\beta), \theta / \beta$, and SE

\begin{tabular}{lllllllll}
\hline & FP & IF & F & T & C & PT & P & O \\
\hline Delta & $/$ & $/$ & $/$ & $/$ & $/$ & $/$ & $/$ & $/$ \\
Theta & $/$ & $/$ & $/$ & $/$ & $/$ & $/$ & $/$ & $/$ \\
Alpha & $/$ & $/$ & 0.001 & $/$ & $<0.001$ & $<0.001$ & $<0.001$ & $<0.001$ \\
Beta & 0.03 & 0.048 & $/$ & $/$ & $/$ & 0.04 & & 0.049 \\
$(\alpha+\theta) / \beta$ & $<0.001$ & $<0.001$ & $<0.001$ & $/$ & $<0.001$ & $<0.001$ & $<0.001$ & $<0.001$ \\
$\alpha / \beta$ & $<0.001$ & $<0.001$ & $<0.001$ & 0.045 & $<0.001$ & $<0.001$ & $<0.001$ & $<0.001$ \\
$(\alpha+\theta) /(\alpha+\beta)$ & $<0.001$ & $<0.001$ & $<0.001$ & $/$ & $<0.001$ & $<0.001$ & $<0.001$ & $<0.001$ \\
$\theta / \beta$ & $<0.001$ & 0.011 & $<0.001$ & $/$ & $<0.001$ & $<0.001$ & $<0.001$ & $<0.001$ \\
SE & $/$ & $/$ & $/$ & $/$ & $/$ & 0.043 & 0.004 & 0.009 \\
\hline
\end{tabular}

Note: shows results with significant p-values $(<0.05)$.

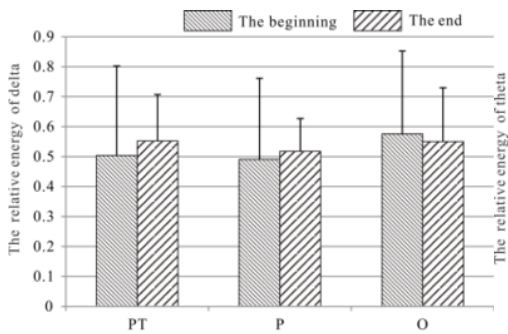

(a)

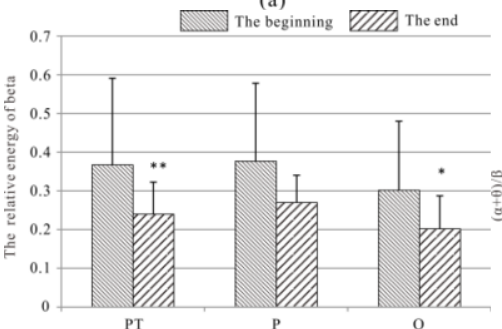

(d)
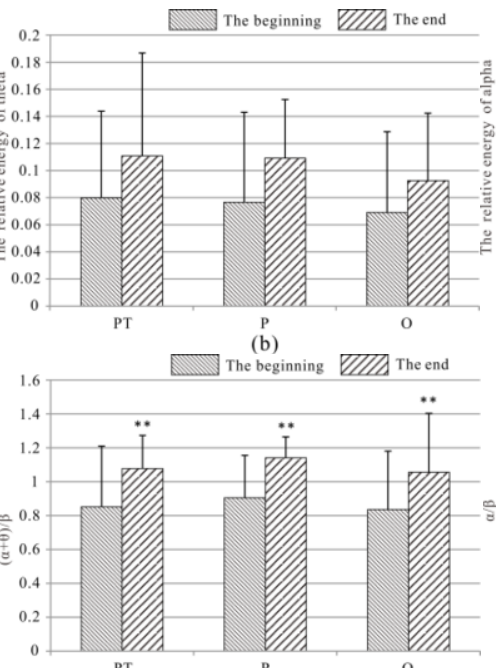

The en

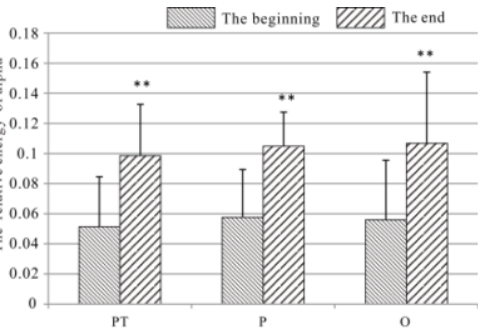

P
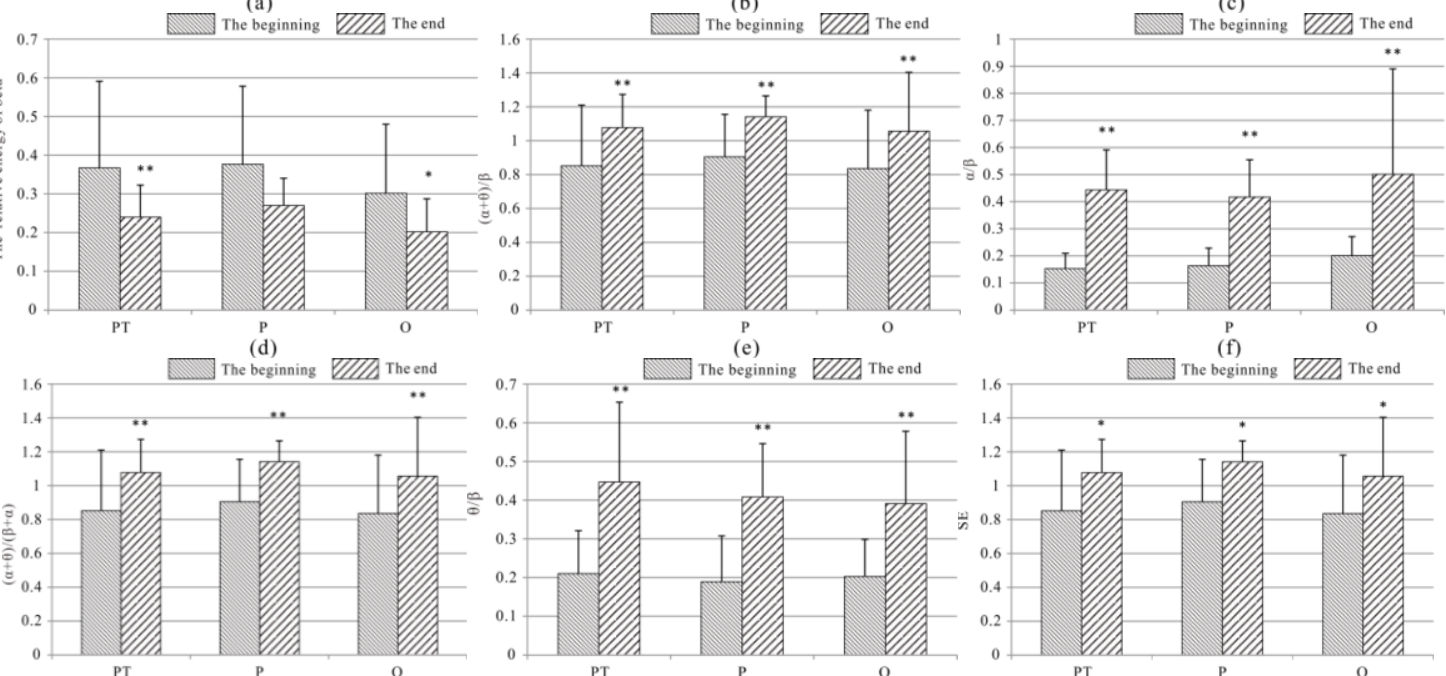

(g)

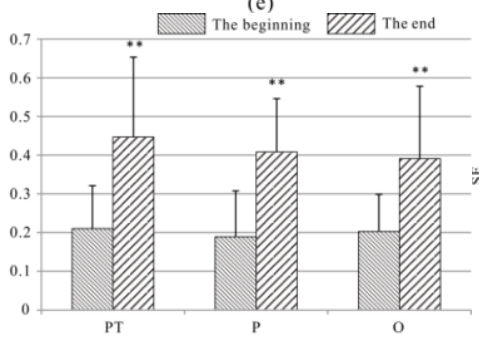

(h)

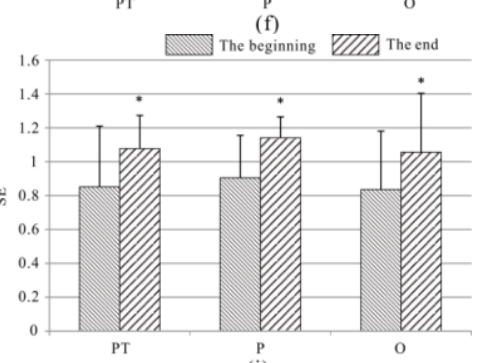

(i)

Fig. 5. Comparison of parameters between the beginning and end of the visual search task.

posterior temporal, parietal, and occipital regions after long-term visual searching. To facilitate the observation of the change trend in all parameters, comparisons of these parameters in the posterior temporal, parietal, and occipital regions between the beginning and end of the task, respectively, are shown in Figure 5. According to Table 2 and Figure 5, we can see that after performing a visual search task, there was a significant increase $(\mathrm{P}<0.05)$ in the relative energy of alpha waves in the frontal, central, posterior temporal, parietal, and occipital lobes; the relative energy in beta waves significantly decreased $(\mathrm{P}<0.05)$ in the pre-frontal, inferior frontal, posterior temporal, and occipital regions; there 

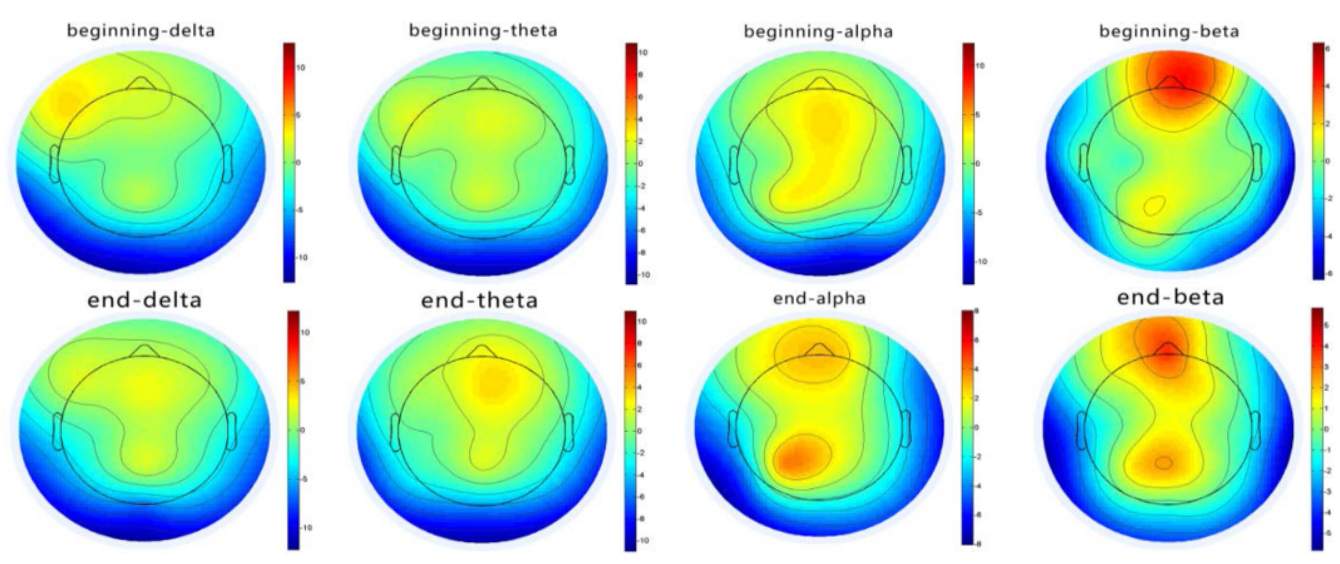

Fig. 6. Brain topography of electroencephalogram activity for a single study subject.

were no significant differences in delta rhythm and theta rhythm in any brain region, but these metrics showed a general increasing trend, shown in Figures 5(a) and 5(b); the four ratio formulas significantly increased $(\mathrm{P}<0.05)$ in all regions except the temporal lobe, wherein only $\alpha / \beta$ changed clearly; and the SE increased $(\mathrm{P}<0.05)$ in the posterior temporal, parietal, and occipital lobes after inducing fatigue.

The brain topography of one subject is shown in Figure 6, which indicates the activities of the four wavebands between the beginning and the end of the task. The blue color indicates low activity, while red indicates high activity. A mild decrease in delta activity occurs in the left of the inferior frontal area. Theta activity has no significant difference in any region except for a slight increase in the frontal region. Alpha activity significantly increased in the frontal, parietal, and occipital lobes. Unlike alpha activity, beta activity was lower in the pre-frontal, frontal, posterior temporal, and occipital areas but was higher in the parietal region at the end of the task.

\section{Discussion}

Fatigue is a complex state manifested by a lack of alertness, weakness, dizziness, or queasiness, which leads to inefficiency and performance reduction. In the present study, ten students participated in the experiment. After performing $60 \mathrm{~min}$ of visual search, RT to stimuli significantly increased, while the accuracy slightly decreased. This reduced task performance is probably because of the appearance of fatigue during the task, which was also verified by subjective evaluation about how each subject perceived his level of fatigue.

EEG measurements have been repeatedly reported to be a reliable method to evaluate mental fatigue. In the current study, our analysis of EEG power spectra revealed that there was a significant increase in alpha activity in the frontal, central, posterior temporal, parietal, and occipital areas, and a dip in beta activity in the pre-frontal, inferior frontal, posterior temporal, and occipital regions at the end of the prolonged visual searching task. Consistently, other studies have also found increasing alpha activity and a drop of beta activity during fatigue [23, 24]. Belyavin and Wright [25] pointed out that the most useful indicator for reduced vigilance was the clear decrease in beta activity, while the increase in alpha activity was proposed as the most sensitive indicator for fatigue by Torsvall and Akerstedt [26]. In addition, Lal and Craig [10] found a significant increase of delta and theta activities during fatigue. However, our study only showed slowly increasing delta and theta activities over time. 
One possible explanation for this discrepancy may be that we used a different definition of frequency band boundaries. Another reason could be that the mental fatigue induced in the present study was too mild for the delta and theta activities to change. Meanwhile, changes in EEG components may be masked by the subjects' motion, such as head movements, as they try to fight fatigue. Considering the reasons above, suitable ratio parameters may be better in distinguishing fatigue level when the variation of basic energy parameters is unclear. Therefore, four formulas, which are denoted as the ratio between the slow and fast wave EEG activities, were calculated in this paper, and all of these ratios increased in all brain regions except the temporal lobe, wherein only $\alpha / \beta$ clearly changed after the visual search task. In comparison with a previous study by Eoh, et al. [27], similar results were obtained for $(\alpha+\theta) / \beta$ and $\alpha / \beta$, which showed an increasing slope. The theta and delta activities had a tendency to slowly increase, so $(\alpha+\theta) /(\alpha+\beta)$ and $\theta / \beta$ also had an increasing slope. The insensitivity of the temporal region to most of these parameters may be because of its irrelevance for vision cognition. Furthermore, the SE significantly increased in the posterior temporal, parietal, and occipital areas, which are consistent with the results from a study by Kar [4]. SE is a measure of flatness of the energy spectrum, and the physical interpretation for this may be understood in the terms of neural activity. When the subjects are performing the visual search task, their attention is more concentrated at the beginning than at the end, which may because of the appearance of fatigue, while the neural activity of the brain was more ordered [28]. Therefore, the value of SE at the beginning of the task was smaller than at the end. In general, the increase in alpha activity, in the four ratios and in SE, as well as the decrease of beta activity, could be used to indicate fatigue in the corresponding brain regions in the present study.

\section{Conclusion}

In the present study, we investigated nine parameters based on the EEG power spectrum in the wavelet domain, in combination with subjective evaluation and reaction time evaluation to assess fatigue induced by a long-term visual search task. All parameters on specific brain regions, except the delta rhythm and theta rhythm, which did not significantly change during fatigue, can be used to indicate a fatigued state.

Although this finding is useful, there are still some potential limitations in the present study. First, the number of study subjects was small, and individual differences can affect the final results. Second, because of the large amount of signal processing and analysis, we only chose to study EEG data from the beginning and end of the visual search task. Moreover, except the subjective evaluation and RT, other measurements such as EOG, EMG and ECG can be used in combination with EEG to study fatigue. All these limitations should be considered in future studies of mental fatigue.

\section{Acknowledgments}

This research was funded by National Natural Science Fund (31170895), National Defense preresearch Fund (A0920132003), and Human Factors Engineering Key Laboratory Fund Project (HF2013-K-06).

\section{References}


[1] E. Waschera, B. Rasch and J. Sänger, Frontal theta activity reflects distinct aspects of mental fatigue, Biological Psychology 96 (2014), 57-65.

[2] D.F. Dinges, An overview of sleepiness and accidents, Journal of Sleep Research 4 (1995), 4-11.

[3] T. Uehata, Karoshi, death by overwork, Nihon Rinsho 63 (2005), 1249-1253.

[4] S. Kar, M. Bhagat and A. Routray, EEG signal analysis for the assessment and quantification of driver's fatigue, Transportation Research Part F 13 (2010), 297-306.

[5] I.D. Brown, Driver fatigue, Human Factors 36 (1994), 298-314.

[6] H.O. Li, J. Seo, K. Kham and S. Lee, Method of measuring subjective 3D visual fatigue: A five-factor model, Digital Holography and Three-Dimensional Imaging, St. Petersburg, Florida, United States, 2008.

[7] J. Gutierrez, P. Perez, F. Jaureguizar and J. Cabrera, Subjective assessment of the impact of the transmission errors in 3DTV compared to HDTV, 3DTV Conference: The True Vision-Capture, Transmission and Display of 3D Video (3DTV-CON), Madrid, Spain, 2011, pp. 1-4.

[8] X.X. Li, H.J. Bi and L.P. Sheng, The EEG changes during night-time driver fatigue, IEEE Transactions on Intelligent Vehicles Symposium, Xi'an, China, 2009, 935-939.

[9] J.P. Liu, C. Zhang and C.X. Zheng, EEG-based estimation of mental fatigue by using KPCA-HMM and complexity parameters, Biomedical Signal Processing and Control 5 (2010), 124-130.

[10] S.K. Lal, A. Crag, P. Brood, L. Kirkup and H. Nguyen, Development of an algorithm for EEG-based drive fatigue countermeasure, Journal of Safety Research 34 (2003), 321-328.

[11] D. Aeschbach, J.R. Matthews, T.T. Postolache, M.A. Jackson, H.A. Giesen and T.A. Wehr, Dynamics of the human EEG during prolonged wakefulness: Evidence for frequency-specific circadian and homeostatic influences, Neuroscience Letters 239 (1997), 121-124.

[12] J. Phipps-Nelson, J.R. Redman and S.M. Rajaratnam, Temporal profile of prolonged, night-time driving performance: Breaks from driving temporarily reduce time-on-task fatigue but not sleepiness, Journal of Sleep Research 20 (2011), 404-415.

[13] C. Zhao, M. Zhao, J. Liu and C. Zheng, Electroencephalogram and electrocardiograph assessment of mental fatigue in a driving simulator, Accident Analysis \& Prevention 45 (2012), 83-90.

[14] J.F. O'Hanlon and G.R. Kelly, Comparison of performance and physiological changes between drivers who perform well and poorly during prolonged vehicular operation, in: Vigilance, R. Mackie, ed., Plenum Press, New York, 1977, pp. $87-109$

[15] J.A. Horne and S.D. Baulk, Awareness of sleepiness when driving, Psychophysiology 41 (2004), 161-165.

[16] D. Waard and K.A. Brookhuis, Assessing driver status: a demonstration experiment on the road, Accident Analysis and Prevention 23 (1991), 297-307.

[17] B. Jap, Using EEG spectral components to assess algorithms for detecting fatigue, Expert Systems with Applications 36 (2009), 2352-2359.

[18] J.F. May and C.L. Baldwin, Driver fatigue: The importance of identifying causal factors of fatigue when considering detection and countermeasure technologies, Transportation Research Part F 12 (2009), 218-224.

[19] T. Jung, S. Makeig, M. Stensmo and T.J. Sejnowski, Estimating alertness from the EEG power spectrum, IEEE Transactions on Biomedical Engineering 44 (1997), 60-69.

[20] C.E. Shannon, A mathematical theory of communication, The Bell Systems Technical Journal 27(1948), 379-423.

[21] J. Lim, W.C. Wu, J. Wang, J.A. Detre, D.F. Dinges and H. Rao, Imaging brain fatigue from sustained mental workload: An ASL perfusion study of the time-on-task effect, NeuroImage 49 (2010), 3426-3435.

[22] J. Lim, R. Ebstein, C.Y. Tse, M. Monakhov, P.S. Lai, D.F. Dinges and K. Kwok, Dopaminergic polymorphisms associated with time-on-task declines and fatigue in the Psychomotor Vigilance Test, PLos One 7 (2012), e33767.

[23] G. Kecklund and T. Akerstedt, Sleepiness in long distance truck driving: An ambulatory EEG study of night driving, Ergonomics 36 (1993), 1007-1017.

[24] J.M. Stern and J. Engel, Atlas of EEG Patterns, Lippincott Williams \& Wilkins, USA, 2005.

[25] A. Belyavin and N.A. Wright, Changes in electrical activity of the brain with vigilance, Electroencephalography and Clinical Neurophysiology 66 (1987), 137-144.

[26] L. Torsvall and T. Akerstedt, Sleepiness on the job: Continuously measured EEG changes in train drivers, Electroencephalography and Clinical Neurophysiology 66 (1987), 502-511.

[27] H.J. Eoh, M.K. Chung and S.H. Kim, Electroencephalographic study of drowsiness in simulated driving with sleep deprivation, International Journal of Industrial Ergonomics 35 (2005), 307-320.

[28] S. Zhang, S.N. Qiao and W. Wang. Melancholia's EEG complexity analysis based on wavelet entropy, Computer Engineering and Applications 48 (2012), 143-145. 